\title{
Antioxidant vitamins $C$ and $E$ affect the superoxide-mediated induction of the soxRS regulon of Escherichia coli
}

\author{
Ana M. Fuentes ${ }^{1}$ and Carlos F. Amábile-Cuevas ${ }^{1,2}$ \\ Author for correspondence: Carlos F. Amábile-Cuevas. Tel: +52 55648146. Fax: +5255648146. \\ e-mail: lusaramex@compuserve.com.mx
}

1 Departamento de Microbiología, LUSARA, Apartado Postal 102-006, 08930 México, D. F., Mexico

2 Departamento de Farmacologla, Facultad de Medicina, UNAM, 04510, México, D. F., Mexico

\begin{abstract}
The mechanism of activation of Escherichia coli redox sensory protein SoxR is still unclear: a [2Fe-2S] cluster contained in a SoxR dimer is potentially redoxsensitive, but the nature of the signal is unknown. Antioxidant vitamins $C$ (ascorbate) and E ( $\alpha$-tocopherol) were used to explore the mechanism of activation of the SoxR protein in vivo. Treating $E$. coli cells with ascorbate or $\alpha$-tocopherol increased their tolerance to paraquat ( $P Q$, a redox-cycling compound), even in the absence of the soxRS locus, suggesting a radicalquenching activity. When using a sox $S^{\prime}:$ : lacz fusion, whose expression is governed by activated SoxR, ascorbate and $\alpha$-tocopherol also prevented the expression of $\beta$-galactosidase after PQ treatment. A secondary activity was observed in cells carrying soxR101, a mutation resulting in the constitutive expression of the sox regulon, where the overexpression of sox $S^{\prime}:$ :lac $Z$ was also reduced by ascorbate or $\alpha$-tocopherol treatment. Additionally, different mechanisms of action were revealed as $\alpha$-tocopherol was capable of preventing both PQ and menadione (MD) lethality, whilst ascorbate prevented PQ lethality but increased MD-mediated cell death. It is proposed that $\alpha$-tocopherol, positioned in membranes, can prevent superoxide-dependent membrane damage; however, water-soluble ascorbate is unable to do so and can even increase the concentration of oxygen radicals reacting with released membrane-associated Fe(II).
\end{abstract}

Keywords: superoxide radical, antioxidants, soxRS, Escherichia coli, vitamins $\mathrm{C}$ and $\mathrm{E}$

\section{INTRODUCTION}

Escherichia coli cells cope with oxidative stress produced by extracellular nitric oxide or intracellular superoxide mainly by the induction of at least 11 genes regulated by the soxRS locus (Demple, 1991). The SoxR protein is responsible for the first regulatory step of a minicascade. It is produced as inactive apo-SoxR and then it is dimerized and two $\mathrm{Fe}-\mathrm{S}$ clusters are assembled into the dimer, possibly in cysteine residues near the $\mathrm{C}$ termini of each monomer (Hidalgo et al., 1995). These centres are susceptible to oxidation, thus stabilizing the dimer and making for a stronger binding of RNA polymerase to the soxS promoter (Hidalgo \& Demple, 1994). Both reduced and oxidized forms of [2Fe2S]SoxR, and even apo-SoxR in vitro, have equal affinity for the soxS promoter, but only [2Fe-2S]SoxR is capable of inducing the transcription of soxS. The likely difference lies in the fact that oxidized [2Fe-2S]SoxR is stable, whereas reduced [2Fe-2S]SoxR is not and may spontaneously revert to the apo-SoxR form incapable of relating to $\sigma^{70}$-containing RNA polymerase (Hidalgo \& Demple, 1996; Hidalgo et al., 1995). On the other hand, recent experiments have shown that reversion to the apo-SoxR form does not occur (Gaudu et al., 1997), so a difference in affinity or a DNA conformational change could be responsible for transcriptional activation by the oxidized but not the reduced form. SoxS, in turn, is responsible for the transcription of around 11 defence and repair genes, while acting as an auto-repressor (Nunoshiba et al., 1992, 1993b).

The nature of the signal that triggers [2Fe-2S]SoxR activation is still unclear; furthermore, most studies on the subject have been done using the purified protein in vitro. Agents or conditions that increase the intracellular concentration of superoxide radicals $\left(\mathrm{O}_{2}^{--}\right)$specifically elicit the soxRS response (Nunoshiba et al., 1992). Exposure to nitric oxide (Nunoshiba et al., 1993a, 1995) 
Table 1. Strains and plasmids

\begin{tabular}{|ll|}
\hline $\begin{array}{l}\text { Strain or } \\
\text { plasmid }\end{array}$ & \\
\hline Strains & \multicolumn{1}{c|}{ Genotype } \\
GC4468 & E. coli $K-12$ soxR $S^{+} \mathrm{F}^{-} \Delta l a c$ rpsL \\
DJ901 & GC4468 $\Delta($ soxRS - zjc2205) zjc2204::Tn10Km \\
JTG1052 & GC4468 soxR101 zjc2206::Tn10Km \\
TN521 & DJ901 $\phi\left(\right.$ soxR $R^{+}$soxS $::$lacZ) \\
TN531 & DJ901 $\phi(\Delta s o x R$ soxS $::$ lacZ) \\
TN5315 & TN531 but recA56 srlC300::Tn10Km \\
Plasmid & \\
pTN101 & pSE380 (Invitrogen) with an EcoRI-HindIII PCR (soxR101) insert \\
\hline
\end{tabular}

and other nitrous compounds (Nunoshiba \& Demple, 1993) also activates SoxR. It has been suggested that direct oxidation of the Fe-S clusters might be the signal (Hidalgo \& Demple, 1994), or that this oxidation might be the consequence of altered $\mathrm{NAD}(\mathrm{P}) \mathrm{H} / \mathrm{NAD}(\mathrm{P})^{+}$or oxidized/reduced 'doxin' (as in flavo-doxins and ferredoxins) ratios (Liochev et al., 1994). Yet another possibility is that the [2Fe-2S] cluster in SoxR is continuously being oxidized under aerobic conditions and being actively reduced by an enzymic step; this putative enzyme could either be superoxide-sensitive or NAD $(\mathrm{P}) \mathrm{H}$-dependent (Hidalgo \& Demple, 1994; Liochev \& Fridovich, 1992; Gaudu et al., 1997). Failure to reduce SoxR results in the accumulation of the oxidized, stable form, leading to the activation of the regulon promoters through SoxS.

In addition to the sox $R S$ response, $\sim 30$ other genes are activated in E. coli cells during superoxide exposure (Demple \& Amábile-Cuevas, 1991). In addition, constitutive enzymes that specifically inactivate superoxide (e.g. Fe- and $\mathrm{Cu}, \mathrm{Zn}$-superoxide dismutases) exist, and nonspecific antioxidant defence mechanisms such as glutathione and glutathione reductase. Antioxidant vitamins, which play definitive roles in higher organisms, do not seem to be important in prokaryotes.

Antioxidant vitamins have been known to act as powerful free radical quenchers in several biological systems through their ability to accept an additional electron and become radicals themselves. These vitamin radicals are more stable, and can be regenerated, avoiding in this way possible cell damage (Moslen, 1992). In higher eukaryotes, ascorbic acid (vitamin C) acts as a free radical quencher in aqueous cell compartments, becoming an ascorbyl radical and then dehydroascorbic acid. $\alpha$-Tocopherol (vitamin E) exerts its antioxidant action in lipophilic cell domains such as membranes. When lipid peroxidation occurs, $\alpha$-tocopherol stops the chain reaction by becoming a cromanyl radical. In this relatively stable radical form, the unpaired electron rotates in $\alpha$-tocopherol's cromanyl ring. The cromanyl radical can be regenerated by two ascorbate molecules or by the glutathione-glutathione reductase system (Ames et al., 1993).
In this work, we treated $E$. coli cells with ascorbate or $\alpha$-tocopherol to ascertain if they can affect the superoxide-dependent activation of the soxRS regulon in vivo; we also assessed the possible protective role of these antioxidant vitamins in superoxide lethality. As ascorbate and $\alpha$-tocopherol are not known to be essential for bacterial metabolism, these experiments also provided important clues as to toxicity mechanisms of redox-cycling compounds used to generate intracellular superoxide and on the physiology of the soxRS response itself.

\section{METHODS}

Strains, media and reagents. E. coli strains used are listed in Table 1 and were kind gifts from B. Demple, Harvard School of Public Health, MA, USA. They were all grown in LB broth or solid media (Maniatis et al., 1982). Paraquat (PQ; Sigma) and menadione bisulfite (MD; Sigma) were used as superoxide-generating agents (Cunningham et al., 1986). Fresh sodium ascorbate (Sigma) solution was added to the indicated concentrations; $\alpha$-tocopherol acetate (a gift from Searle de México) was added as an homogeneous suspension, prepared in a tissue grinder just before use.

$P Q, M D$, ascorbate and $\alpha$-tocopherol treatment in liquid media. Overnight bacterial cultures of strains TN521 and TN531 were diluted and grown to early exponential phase in LB broth at $37^{\circ} \mathrm{C}$ in an orbital shaker (150 r.p.m.); ascorbate to final concentrations of $0.1,1.0$ and $10.0 \mathrm{mM}$, or $\alpha$-tocopherol to $0 \cdot 01,0 \cdot 1,1 \cdot 0$ and $10.0 \mathrm{mM}$ were added and the mixtures incubated for $15 \mathrm{~min}$. Twenty-five or $50 \mu \mathrm{M} P Q$ or $300 \mu \mathrm{M} M \mathrm{MD}$ was then added, followed by further incubation for $30 \mathrm{~min}$ before measurement of $\beta$-galactosidase activity. Strain TN5315/pTN101 was grown to early exponential phase and then IPTG (Sigma) was added to a concentration of $1 \mathrm{mM}$. After $30 \mathrm{~min}$, ascorbate or $\alpha$-tocopherol $(10 \mathrm{mM})$ was added and cells incubated for another $15 \mathrm{~min}$, at the end of which superoxide-generating agents were added. After a $30 \mathrm{~min}$ incubation, $\beta$-galactosidase activity was assayed.

Measurement of $\beta$-galactosidase activity. This was done as described by Miller (1992). Briefly, the optical density (at $600 \mathrm{~nm}$ ) of each culture was determined and cells permeated with SDS/chloroform (because of coloration produced by $\mathrm{MD}$, cultures treated with this agent were washed by centrifugation and resuspension before $\mathrm{OD}_{600}$ determination); an aliquot was added to $\mathrm{Z}$ buffer $\left(\mathrm{Na}_{2} \mathrm{HPO}_{4} .7 \mathrm{H}_{2} \mathrm{O}, 60 \mathrm{mM}\right.$; $\mathrm{NaH}_{2} \mathrm{PO}_{4} . \mathrm{H}_{2} \mathrm{O}, 40 \mathrm{mM} ; \mathrm{KCl}, 12 \mathrm{mM} ; \mathrm{MgSO}_{4} .7 \mathrm{H}_{2} \mathrm{O}$, 
$1 \mathrm{mM} ; \beta$-mercaptoethanol, $38.5 \mathrm{mM} ; \mathrm{pH} 7 \cdot 0)$ containing ONPG (Sigma), the mixture incubated at $37^{\circ} \mathrm{C}$ until a change in colour was detected and optical density then measured at $420 \mathrm{~nm}$.

Preparation of PQ and MD gradient plates with and without vitamins. To assess the effect of ascorbate and $\alpha$-tocopherol on $P Q$ and $M D$ lethality, $P Q /$ vitamin gradient plates were prepared as reported previously (Cunningham et al., 1986). LB agar plates contained PQ [14 mg $\left.(40 \mathrm{ml})^{-1}\right]$ or $\mathrm{MD}$ [90 mg $\left.(40 \mathrm{ml})^{-1}\right]$ on the bottom, and ascorbate or $\alpha$-tocopherol $(10 \mathrm{mM})$ on the top. Early-exponential-phase liquid cultures of strains GC4468 and DJ901 were suspended in liquefied soft LB agar and 'stamped' across the gradient using a glass slide. Plates were incubated at $37^{\circ} \mathrm{C}$ for $18 \mathrm{~h}$ and the length of growth was then measured. Strain JTG1052 was assayed in the same way, but plates contained $28 \mathrm{mg} P Q(40 \mathrm{ml})^{-1}$ to achieve measurable killing of this oxidative-stress resistant strain; MD concentration was the same as above.

\section{RESULTS}

\section{Ascorbate and $\alpha$-tocopherol inhibit PQ-mediated induction of soxS': :lacZ}

PQ treatment of strain TN521 induced the expression of the sox $S^{\prime}:$ : lac $Z$ fusion, measured as increased $\beta$-galactosidase activity compared to basal levels (1.5 to 2 - and 3 to 4-fold induction by 25 or $50 \mu \mathrm{M}$ PQ treatment, respectively; Table 2 ). This induction was prevented in a dose-dependent manner by ascorbate and $\alpha$-tocopherol treatment, although lower concentrations of $\alpha$-tocopherol were required to achieve the same effects as for ascorbate. Basal levels were also diminished in the cells treated only with $10 \mathrm{mM}$ ascorbate or $\alpha$-tocopherol (Table 2). Strain TN531 (soxR) was unresponsive to either PQ or vitamin treatments (data not shown).

\section{$\alpha$-Tocopherol inhibits MD-mediated induction of soxS': lacZ but ascorbate does not}

In strain TN521, the increase in $\beta$-galactosidase activity induced by $300 \mu \mathrm{M} \mathrm{MD}$ treatment was similar to that obtained with $50 \mu \mathrm{M}$ PQ ( 3 -fold; Table 2); $\alpha$-tocopherol also prevented the induction in a dosedependent manner (Table 2) but ascorbate showed a diminished preventive activity, lower as its concentration increased. Experiments with strain TN531 also proved that these effects were SoxR-dependent.

\section{A sox $R^{c}$ mutant is also responsive to vitamin treatments}

Ascorbate and $\alpha$-tocopherol were capable of reducing $\beta$-galactosidase activity in strain TN5315/pTN101 both in IPTG-treated and non-induced cells. Plasmid pTN101 carries a copy of soxR101, a sox $R$ allele that mediates the constitutive expression of the sox regulon genes (thus named sox $R^{\mathrm{c}}$ ), even in the absence of oxidative stress. This constitutive expression was diminished by antioxidants, $27 \%$ by ascorbate and $50 \%$ by $\alpha$-tocopherol. Treatment with IPTG elicited a $\sim 2 \cdot 2$-fold overinduction of the $s o x S^{\prime}:$ : lacZ fusion (actual levels of Sox $\mathrm{R}^{\mathrm{c}}$ were not assessed), which was also reduced by ascorbate $(31 \%)$ and $\alpha$-tocopherol $(53 \%)$ treatment.

\section{Protective effects of ascorbate and $\alpha$-tocopherol on PQ-induced lethality}

PQ lethality was prevented completely by the presence of $10 \mathrm{mM} \alpha$-tocopherol, in both GC4468 (soxR $S^{+}$) and DJ901 ( $\Delta$ soxRS) strains; $10 \mathrm{mM}$ ascorbate totally protected GC4468 cells but protected only partially DJ901 cells (Fig. 1). Dehydroascorbate had no effect on cell killing by PQ (data not shown). Strain JTG1052 (soxR101), a constitutively active SoxR mutant, was resistant to $\mathrm{PQ}$ concentrations used against strains GC4468 and DJ901; it was slightly protected by both vitamins against a double PQ concentration (Fig. 1).

\section{Effects of ascorbate and $\alpha$-tocopherol on MD-induced lethality}

Cell killing was completely prevented with $\alpha$-tocopherol in strains JTG1052 (soxR101), GC4468 (soxR $S^{+}$) and DJ901 ( $\Delta$ soxRS). Surprisingly, ascorbate not only did

Table 2. Effect of oxidants and antioxidants on strain TN521 (harbouring soxS': :lacZ fusion)

Values represent $\beta$-galactosidase activity compared to the basal level $(\equiv 1)$ and are means of at least three individual experiments. Standard deviations were no greater than $10 \%$ in any case. ND, Not determined.

\begin{tabular}{|c|c|c|c|c|c|c|c|c|}
\hline \multirow[t]{3}{*}{ Oxidant $(\mu \mathrm{M})$} & \multicolumn{8}{|c|}{ Antioxidant (mM) } \\
\hline & \multirow[t]{2}{*}{ None } & \multicolumn{3}{|c|}{ Ascorbate } & \multicolumn{4}{|c|}{$\alpha$-Tocopherol } \\
\hline & & $0 \cdot 1$ & 1 & 10 & 0.01 & $0 \cdot 1$ & 1 & 10 \\
\hline None & $1 \cdot 0$ & ND & ND & 0.64 & ND & ND & ND & 0.65 \\
\hline PQ 25 & $1 \cdot 78$ & $1 \cdot 26$ & $1 \cdot 02$ & $0 \cdot 75$ & 1.59 & 0.90 & 0.73 & 0.69 \\
\hline PQ 50 & $3 \cdot 0$ & 1.02 & 0.85 & 0.55 & $1 \cdot 60$ & 0.89 & 0.69 & 0.52 \\
\hline MD 300 & $2 \cdot 98$ & 1.02 & $1 \cdot 29$ & 1.93 & 1.73 & $1 \cdot 62$ & 1.09 & 0.81 \\
\hline
\end{tabular}




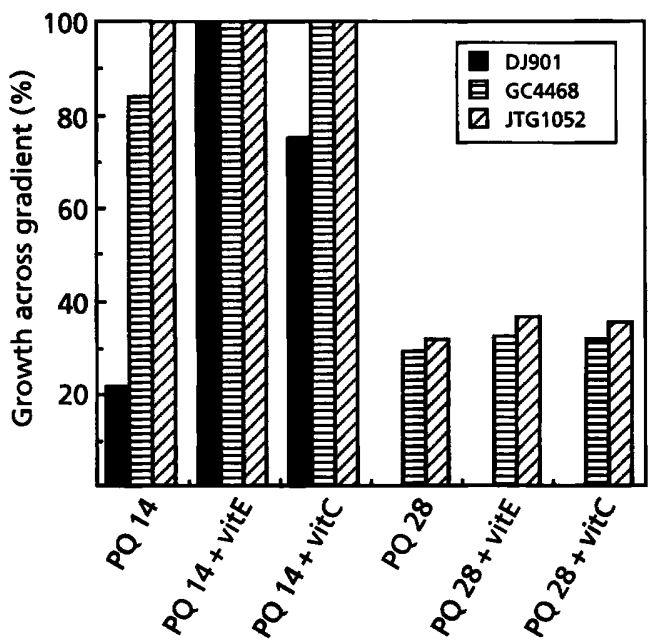

Fig. 1. Effect of antioxidant vitamins on $P Q-$ mediated cell death. Gradient plates containing $P Q$ [14 or $28 \mathrm{mg}(40 \mathrm{ml})^{-1}$ ] were prepared and inoculated as described in Methods. Growth was scored as the percentage of the maximum length possible $(80 \mathrm{~mm}) . \alpha$-Tocopherol (vitE) or ascorbate (vitC) were added to the top layer at a final concentration of $10 \mathrm{mM}$. Strain DJ901 was not assayed on PQ 28 plates. Bars represent results from a typical experiment; at least three independent experiments were performed, with similar results.



Fig. 2. Effect of antioxidant vitamins on MD-mediated cell death. Gradient plates containing MD [90 mg $\left.(40 \mathrm{ml})^{-1}\right]$ were prepared and inoculated as described in Methods. $\alpha$-Tocopherol (vitE), ascorbate (vitC) or dehydroascorbate (DvitC) were added to the top layer at a final concentration of $10 \mathrm{mM}$. Bars represent typical results, from one among at least three experiments.

not protect as effectively as $\alpha$-tocopherol against MD toxicity, but also acted synergically with $\mathrm{MD}$, killing the three strains completely. In contrast, dehydroascorbate did not have such a potent effect on MD-mediated cell killing (Fig. 2).

\section{DISCUSSION}

A large amount of biochemical and molecular evidence on the mechanisms of redox-sensing and activation of the $E$. coli SoxR protein have accumulated recently (Gaudu \& Weiss, 1996; Hidalgo \& Demple, 1994, 1996; Hidalgo et al., 1995; Wu et al., 1995). However, very few in vivo experiments have been done on this subject.

Ascorbate and $\alpha$-tocopherol are known free-radical scavengers (Ames et al., 1993). We observed an increased tolerance to $\mathrm{PQ}$, a water-soluble intracellular superoxide generator, in the presence of both ascorbate and $\alpha$-tocopherol, and a reduction in basal and PQ-induced $\beta$-galactosidase activity in cells bearing the $\operatorname{sox} S^{\prime}:: \operatorname{lac} Z$ fusion when treated with either antioxidant vitamins. However, when using MD, which diverts electrons from the respiratory chain within the cell membrane (Cadenas, 1989), as redox-cycling agent a different effect was observed. Whilst liposoluble $\alpha$-tocopherol prevented both $M D$ toxicity and sox $S^{\prime}:: l a c Z$ induction, hydrosoluble ascorbate increased $M D$ toxicity and poorly prevented $\beta$-galactosidase overproduction in an inversely dose-dependent manner. We propose that $\alpha$-tocopherol quenches radicals generated close to the cell membrane and/or stabilizes the membrane itself, therefore reducing the deleterious effects of both PQ and $M D$. On the other hand, ascorbate is capable of scavenging PQ-generated superoxide in the cytosol, but is less capable of protecting against the effects of $\mathrm{MD}$ within lipophilic environments. Furthermore, as iron availability within the cell seems to be partially regulated by several membrane-bound systems (Hassan \& Schrum, 1994), MD-mediated membrane damage, such as lipoperoxidation, may cause iron release, allowing for the generation of additional oxygen radicals by ascorbate-mediated Fenton reactions (Shamberger, 1984). Fe(II) pretreatment increased by $\sim 10 \%$ the toxicity of $\mathrm{PQ}$ in the presence of ascorbate in gradient plates (unpublished results), suggesting $\mathrm{Fe}$-ascorbate interactions leading to toxic by-products. If these hypotheses are correct, the impact of membrane-related events upon intracellular superoxide toxicity (e.g. lipoperoxide generation, $\mathrm{Fe}(\mathrm{II})$ release, etc.) and soxRS regulatory mechanisms should be examined.

Wild-type SoxR protein has a DNA-binding domain, possibly responsible for binding to the soxS promoter, and a cysteine cluster at the $\mathrm{C}$ terminus (AmábileCuevas \& Demple, 1991), possibly involved in the anchoring of the [2Fe-2S] complex (Hidalgo et al., 1995). Mutations or deletions at the end of the soxR gene (not involving the cysteine cluster), such as those found in soxR101 and JTG1052, result in the readily oxidized but still superoxide-inducible expression of soxS (Nunoshiba \& Demple, 1994; Tsaneva \& Weiss, 1990). Surprisingly, both ascorbate and $\alpha$-tocopherol were able to diminish the $\beta$-galactosidase activity in soxR101, sox $S^{\prime}:$ : lacZ cells, induced only by SoxR 101 overproduction. It is possible that this reflects the radical-quenching capabilities of both antioxidants, since SoxR101 still retains some of its redox-respon- 
siveness. However, we would expect this effect to be equimolar, not proportional (i.e. the reduction in $\beta$ galactosidase Miller units should be the same if antioxidants are just quenching oxygen radicals); but, while the $\beta$-galactosidase activity reduction was of $\sim 12000$ Miller units in TN5315/pTN101-treated cells, it only amounted to $\sim 3000$ units in strain TN521. We surmise that vitamins are acting on SoxR-activating steps apart from the mere quenching of superoxide radicals.

The SoxR protein is obviously generated as a metal-free monomer and may become dimerized even in the absence of iron. A first regulatory step on SoxR activation would be iron availability and, when the [2Fe-2S]-containing dimer is formed, it is readily oxidized to an active, stable form under normal aerobic conditions (Hidalgo et al., 1995). A second regulatory step may involve the active reduction of $[2 \mathrm{Fe}-2 \mathrm{~S}] \mathrm{SoxR}$ back to its unstable form, perhaps through a NAD $(\mathrm{P}) \mathrm{H}$ mediated reaction, catalysed by an unknown enzyme or even by the C-terminal region of SoxR (Hidalgo \& Demple, 1996). NAD(P)H cell pools might be depleted as a consequence of redox stress, preventing SoxR reduction (Liochev \& Fridovich, 1992); also, mutant protein SoxR101 may not be susceptible to reduction due to the mutation near the C-terminus and therefore accumulates in its oxidized form, continuously activating the soxS promoter (Hidalgo et al., 1997). Ferredoxin oxidoreductase, a recently discovered member of the soxRS regulon, acts as a diaphorase during PQ induction but when superoxide production ceases, it acts as a doxin reducer and could be responsible for Sox $\mathrm{R}$ inactivation, acting as a reduction-dependent feedback mechanism of the regulon (Liochev et al., 1994).

$\alpha$-Tocopherol may be capable of preventing SoxR and SoxR101 activation by quenching radicals and protecting membranes from oxidation, therefore diminishing the release of membrane-bound iron. Ascorbate is also capable of scavenging radicals in hydrophilic compartments and may be able to directly reduce oxidized SoxR proteins, returning them to their inactive form. Ascorbate-mediated reduction may be nonspecific, affecting both SoxR and SoxR101 proteins. Glutathione, another water-soluble antioxidant agent, is also capable of affecting SoxR activation in vivo (although it activates SoxR in an oxygen-dependent manner in vitro; Din \& Demple, 1996).

Nitric oxide is another known inducer of the soxRS response and is capable of destroying $\mathrm{Fe}-\mathrm{S}$ clusters in many proteins other than SoxR. $\mathrm{NO}^{\circ}$ is also known to participate in the regulation of iron availability in mammals during infectious processes, although the regulatory effect involves iron sequestering instead of liberation (Sellers et al., 1996). Iron availability affects the expression of at least one member of the soxRS regulon, fumC, in a soxR-dependent way (Park \& Gunsalus, 1995). Since iron ions seem to play a key role in the regulation of the soxRS system, it is likely that antioxidant vitamins are affecting the availability of this metal as well, resulting in the effects described here. Along with the involvement of soxRS in multiple antibiotic resistance, which may even be triggered by environmental pollutants (Fuentes \& Amábile-Cuevas, 1997), its response to iron levels underscores the notion that soxRS may be substantially important in Gramnegative bacterial virulence.

\section{ACKNOWLEDGEMENTS}

We thank B. Demple for the kind gifts of strains and for the free exchange of data. Work with $\alpha$-tocopherol received partial support from Searle de México. This work was supported in part by a Fogarty International Research Collaboration Award to B. Demple and C.F.A.-C.

\section{REFERENCES}

Amábile-Cuevas, C. F. \& Demple, B. (1991). Molecular characterization of the soxRS genes of Escherichia coli: two genes control a superoxide stress regulon. Nucleic Acids Res 19, 4479-4484.

Ames, B. N., Shigenaga, M. K. \& Hagen, T. M. (1993). Oxidants, antioxidants, and the degenerative diseases of aging. Proc Natl Acad Sci USA 90, 7915-7922.

Cadenas, E. (1989). Biochemistry of oxygen toxicity. Annu Rev Biochem 58, 79-110.

Cunningham, R. P., Saporito, S., Spitzer, S. G. \& Weiss, B. (1986). Endonuclease IV ( $n f o$ ) mutant of Escherichia coli. J Bacteriol 168, 1120-1127.

Demple, B. (1991). Regulation of bacterial oxidative stress genes. Annu Rev Genet 25, 315-337.

Demple, B. \& Amábile-Cuevas, C. F. (1991). Redox redux: the control of oxidative stress responses. Cell 67, 1-20.

Din, H. \& Demple, B. (1996). Glutathione-mediated destabilization in vitro of $[2 \mathrm{Fe}-2 \mathrm{~S}]$ centers in the SoxR regulatory protein. Proc Natl Acad Sci USA 93, 1792-1796.

Fuentes, A. M. \& Amábile-Cuevas, C. F. (1997). Mercury induces multiple antibiotic resistance in Escherichia coli through activation of SoxR, a redox-sensing regulatory protein. FEMS Microbiol Lett 154, 385-388.

Gaudu, P. \& Weiss, B. (1996). SoxR, a [2Fe-2S] transcription factor, is active only in its oxidized form. Proc Natl Acad Sci USA 93, 10094-10098.

Gaudu, P., Moon, N. \& Weiss, B. (1997). Regulation of the soxRS oxidative stress regulon. Reversible oxidation of the $\mathrm{Fe}-\mathrm{S}$ centers of SoxR in vivo. J Biol Chem 272, 5082-5086.

Hassan, H. M. \& Schrum, L. W. (1994). Roles of manganese and iron in the regulation of the biosynthesis of manganese-superoxide dismutase in Escherichia coli. FEMS Microbiol Rev 14, 315-324.

Hidalgo, E. \& Demple, B. (1994). An iron-sulfur center essential for transcriptional activation by the redox-sensing SoxR protein. EMBO J 13, 138-146.

Hidalgo, E. \& Demple, B. (1996). Activation of SoxR-dependent transcription in vitro by noncatalytic or NifS-mediated assembly of [2Fe-2S] clusters into apo-SoxR. J Biol Chem 271, 7269-7272.

Hidalgo, E., Bollinger, J. M., Bradley, T. M., Walsh, C. T. \& Demple, B. (1995). Binuclear [2Fe-2S] clusters in the Escherichia coli SoxR protein and role of the metal centers in transcription. J Biol Chem 270, 20908-20914. 
Hidalgo, E., Ding, H. \& Demple, B. (1997). Redox signal transduction: mutations shifting [2Fe-2S] centers of the SoxR sensor-regulator to the oxidized form. Cell 88, 121-129.

Liochev, S. I. \& Fridovich, I. (1992). Fumarase C, the stable fumarase of Escherichia coli, is controlled by the soxRS regulon. Proc Natl Acad Sci USA 89, 5892-5896.

Liochev, S. I., Hausladen, A., Beyer, W. F. \& Fridovich, I. (1994). NADPH: ferredoxin oxidoreductase acts as a paraquat diaphorase and is a member of the soxRS regulon. Proc Natl Acad Sci USA 91, 1328-1331.

Maniatis, T., Fritsch, E. F. \& Sambrook, J. (1982). Molecular Cloning: a Laboratory Manual. Cold Spring Harbor, NY: Cold Spring Harbor Laboratory.

Miller, J. H. (1992). A Short Course in Bacterial Genetics. Cold Spring Harbor, NY: Cold Spring Harbor Laboratory.

Moslen, M. T. (1992). Protection against free radical-mediated injury. In Free Radical Mechanisms of Tissue Injury, pp. 203-216. Edited by M. T. Moslen \& C. V. Smith. New York: CRC Press. Nunoshiba, T. \& Demple, B. (1993). Potent intracellular oxidative stress exerted by the carcinogen 4-nitroquinoline- $N$-oxide. Cancer Res 55, 3250-3252.

Nunoshiba, T. \& Demple, B. (1994). A cluster of constitutive mutations affecting the C-terminus of the redox-sensitive SoxR transcriptional activator. Nucleic Acids Res 22, 2958-2962.

Nunoshiba, T., Hidalgo, E., Amábile-Cuevas, C. F. \& Demple, B. (1992). Two-stage control of an oxidative stress regulon: the Escherichia coli SoxR protein triggers redox-inducible expression of the soxS regulatory gene. J Bacteriol 174, 6054-6060.

Nunoshiba, T., DeRojas-Walker, T., Wisshnok, J. S., Tannenbaum, S. R. \& Demple, B. (1993a). Activation by nitric oxide of an oxidative-stress response that defends Escherichia coli against activated macrophages. Proc Natl Acad Sci USA 90, 9993-9997.

Nunoshiba, T., Hidalgo, E., Li, Z. \& Demple, B. (1993b). Negative autoregulation by the Escherichia coli SoxS protein: a dampening mechanism for the sox $R S$ redox stress response. J Bacteriol 175, 7492-7494.

Nunoshiba, T., DeRojas-Walker, T., Tannenbaum, S. R. \& Demple, B. (1995). Roles of nitric oxide in inducible resistance of Escherichia coli to activated murine macrophages. Infect Immun 63, 794-798.

Park, S.-J. \& Gunsalus, R. P. (1995). Oxygen, iron, carbon, and superoxide control of the fumarase fumA and fum $\mathrm{C}$ genes of Escherichia coli: role of arcA, fnr and soxR gene products. J Bacteriol 177, 6255-6262.

Sellers, V. M., Johnson, M. K. \& Dailey, H. A. (1996). Function of the $[2 \mathrm{Fe}-2 \mathrm{~S}]$ cluster in mammalian ferrochelatase: a possible role as a nitric oxide sensor. Biochemistry 35, 2699-2704.

Shamberger, R. J. (1984). Genetic toxicology of ascorbic acid. Mutat Res 133, 135-159.

Tsaneva, I. R. \& Weiss, B. (1990). soxR, a locus governing a superoxide response regulon in Escherichia coli K-12. J Bacteriol 172, 4197-4205.

Wu, J., Dunham, W. R. \& Weiss, B. (1995). Overproduction and physical characterization of SoxR, a $[2 \mathrm{Fe}-2 \mathrm{~S}]$ protein that governs an oxidative response regulon in Escherichia coli. J Biol Chem 270, 10323-10327.

Received 15 December 1997; revised 20 February 1998; accepted 10 March 1998. 